\title{
Picosecond Laser Direct Patterning of Poly(3,4-ethylene dioxythiophene)-Poly(styrene sulfonate) (PEDOT:PSS) Thin Films
}

\author{
Shizhou XIAO, Susana ABREU FERNANDES, Cemal ESEN, Andreas OSTENDORF \\ Applied Laser Technologies, Ruhr University Bochum, 44801 Bochum, Germany \\ Email:xiao@lat.rub.de
}

\begin{abstract}
Laser selective micro patterning of thin film of Poly (3,4-ethylenedioxythiophene):Poly (styrene sulfonate) (PEDOT:PSS) blend is investigated. Picosecond pulsed laser with $355 \mathrm{~nm}$ and $1064 \mathrm{~nm}$ wavelengths are used to study the ablation behavior of PEDOT:PSS thin film coated on glass. We present and discuss ablation thresholds for different film thicknesses as well as ablation lines with different overlapping rates. The results observed by SEM and white-light interference microscopy reveal that PEDOT:PSS film on glass substrates can be selectively patterned by optimized laser parameters.
\end{abstract}

DOI:10.2961/jlmn.2011.03.0015

Keywords: laser direct patterning, PEDOT:PSS conducting polymer, flexible electronics, thin film ablation, organic photovoltaics.

\section{Introduction}

PEDOT:PSS, a polymer blend that consists of a conducting poly(3,4-ethylenedioxythiophene) polycation (PEDOT) and a poly(styrenesulfonate) polyanion (PSS), is extensively used as electrode material in flexible electronics applications such as sensors, memories, organic photovoltaic (OPV) and organic light-emitting diodes (OLEDs). The benefits from this blend is not only due to its high conductivity $(>200 \mathrm{~S} / \mathrm{cm})$, high transmission in visible spectrum as a thin film, but also for its water-soluble property. This means highly transparent and conductive surface coatings can be prepared by spin coating or slot-die coating on almost any hydrophilic surface, compatible to the high speed roll-to-roll mass production line. It is very attractive for decreasing the price for future flexible electronic devices, also as a replacement of the traditionally used indium tin oxide (ITO), which is becoming more and more expensive.

Flexible electronic devices are mostly realized by a multilayer design with special patterns on each layer and sophisticated alignment. Unfortunately, most solution based coating methods used in roll-to-roll process are not compatible with the downsizing of features to microns or even sub microns scale and, consequently, lead to the loss of valuable area. Therefore additional patterning processes are necessary. Several methods may be able to achieve small feature sizes for coated PEDOT:PSS thin film, such as lithography [1], micro contact printing [2-4], plasma patterning [5], laser induced forward transfer [6] and laser direct patterning [7-8] technologies. Among these methods, laser direct patterning technology is the most prospective candidate due to its flexibility, fast speed and most important, compatibility to roll-to-roll process. Recently there are some publications discussing this topic. In ref. [9], the authors show multi-beam patterning lines generated by multi beam interference with 150 fs femtosecond laser system in PEDOT:PSS. In ref. [8], the authors used 1064 $\mathrm{nm}$ picosecond laser patterning of PEDOT:PSS thin films and provided some discussion about the Raman spectrum change of the material after the laser processing. In ref. [7] the authors used the excimer lasers at $193 \mathrm{~nm}$ and $248 \mathrm{~nm}$ wavelength to determine the ablation threshold and demonstrate some areal patterning examples. In this paper we investigate and compare the picosecond laser patterning results at $355 \mathrm{~nm}$ and $1064 \mathrm{~nm}$ wavelength, as well as multi pulse ablation, also the overlapping rate behavior will be discussed.

\section{Experimental details}

\subsection{Thin film preparation}

In our experiments we used a commercially available PEDOT:PSS blend (H.C. Stark, type P510). In order to produce the desired thin film, we used spin coating on the cover glass for lab scale purpose with different spin speed and solid content of PEDOT:PSS solution. After spin coating, the sample was heated up to $120^{\circ} \mathrm{C}$ for $10 \mathrm{~min}$ to evaporate the water and anneal the PEDOT:PSS material. After drying the layer thickness was measured by whitelight interference (Polytec Inc.). Surface resistance of the thin film was determined by four-point-probe method (Keithley SourceMeter 2400). The transmission spectrum was detected by UV-Vis spectrometer (V-660, Jasco Inc.).

\subsection{Laser ablation system}

The experimental set-up included a pulsed Nd:YAG laser with a fundamental wavelength of $1064 \mathrm{~nm}$ and the third harmonic wavelength $355 \mathrm{~nm}$. The pulse duration was $10 \mathrm{ps}$ and the repetition rate was set to $200 \mathrm{kHz}$ (Lumera Hyperrapid 25). The Gaussian beam diameter was $10 \mathrm{~mm}$ behind the beam expander, with an $\mathrm{M}^{2}$ quality parameter of 1.3. Galvanometric scanners (HurryScan14 for $1064 \mathrm{~nm}$, HurryScan 10 for $355 \mathrm{~nm}$, Scanlab $\mathrm{GmbH}$ ) equipped with F-theta lenses (LINOS) were used to move the beam 
relative to the sample. A pulse train was generated by controlling the open time of external electro-optic modulator (EOM), which is capable of switching repetition rates from 0 up to $200 \mathrm{kHz}$. The whole system diagram is shown in figure 1 .

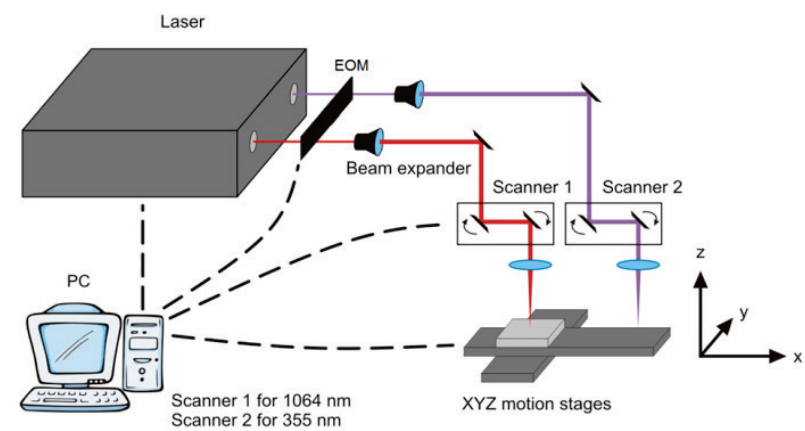

Fig 1. The schematic diagram of laser ablation system

\section{Results and Discussion}

\subsection{Sample preparation}

The aqueous solution of PEDOT:PSS was spin coated on the cover glass. Figure 2 illustrates how the turning speed of the spin-coater and the PEDOT:PSS solid content affect the coated film thickness. By decreasing the solid content, the viscosity of aqueous PEDOT:PSS blend also decreases resulting in thinner films.

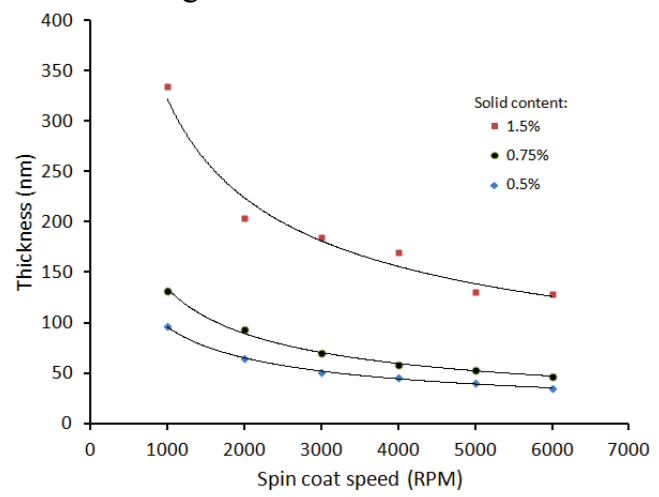

Fig 2. The PEDOT:PSS film thickness varies with different spin-coat velocities and solid content. The solid lines are used for guiding eyes only.

The film thickness has an important effect on the device performance due to the surface resistance changes following the film thickness as shown in figure 3[10]. With the thicker film better electrical conductivity can be obtained. As the film thickness falls below $100 \mathrm{~nm}$, the resistance strongly increases.

The optical spectral transmittance of $100 \mathrm{~nm}$ thick PEDOT:PSS coated on glass was measured by UV-VIS spectrophotometer. The influence from the glass substrate was subtracted, resulting in the transmission spectrum of PEDOT:PSS film shown in figure 4. PEDOT:PSS holds good transmission in the visible wavelength range. High absorption occurs at the near infrared region. In this paper we used $355 \mathrm{~nm}$ and $1064 \mathrm{~nm}$ wavelengths laser pulses for structuring.

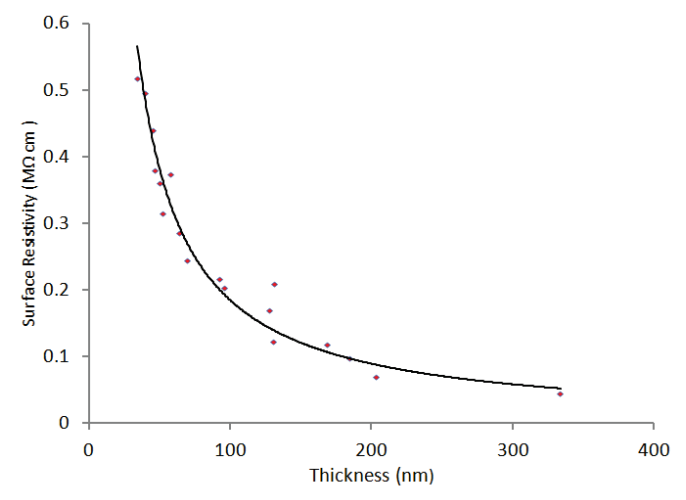

Fig 3. The surface resistivity of the PEDOT:PSS film varies with the film thickness. Four point-probe measurements have been performed on PEDOT:PSS films in-plane across a $1 \mathrm{~mm}$ channel.

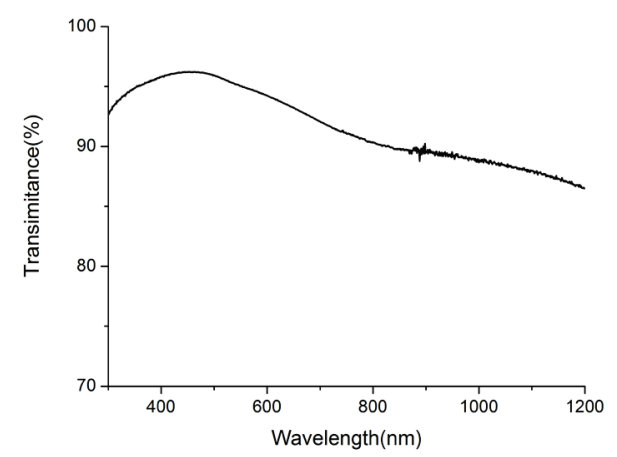

Fig 4. The transmission spectrum of $100 \mathrm{~nm}$ thick PEDOT:PSS film measured by UV-Vis spectrophotometer. The curve was subtracted of the effect induced by the glass substrate.

\subsection{Single-pulse experiments}

Due to the different absorption behavior of the material, several laser/matter interactions mechanisms exist, such as thermal evaporation, photochemical reaction, photomechanical punching and so on. In ref. [8] the authors demonstrate that the picoseconds laser ablation of PEDOT:PSS film by $1064 \mathrm{~nm}$ wavelength is mainly caused by a photomechanical force. In our experiments we studied both the frontside and backside laser irradiation. The different directions of the incident beam induced different thermal distributions within the film, especially in thick film. The gradient of the temperature field induced a different stress distribution which changes the rim shape around the spot. In this section we study the frontside and backside ablation effect using $355 \mathrm{~nm}$ and $1064 \mathrm{~nm}$ wavelengths on 100 nm PEDOT:PSS coated samples.

The single pulse ablation results using $355 \mathrm{~nm}$ wavelength are shown in figure 5. The PEDOT:PSS film could be selectively removed using backside and frontside illumination. In the low fluence area the backside ablation provided better performance demonstrating a uniform and clear rim of the spot. However, the rim got higher quality for frontside irradiation by increasing the laser fluence. The backside irradiation accumulated more laser energy around the interface of the film and the glass, which induced higher 
temperatures and caused glass substrate damage with lower fluence comparing to frontside ablation. Also relatively high absorption of the glass at $355 \mathrm{~nm}$ wavelength aggravates the damage to the glass.

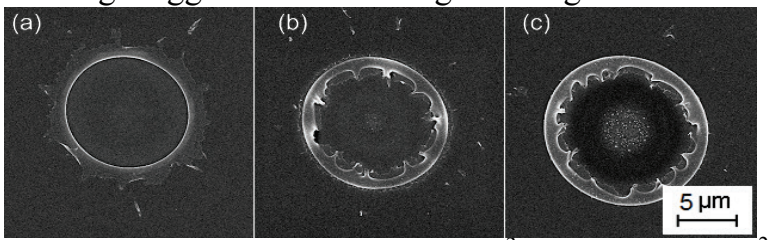

Backside irradiation: (a). $\mathrm{f}=0.79 \mathrm{~J} / \mathrm{cm}^{2}$; (b). $\mathrm{f}=0.92 \mathrm{~J} / \mathrm{cm}^{2}$; (c). $\mathrm{f}=1.06 \mathrm{~J} / \mathrm{cm}^{2}$

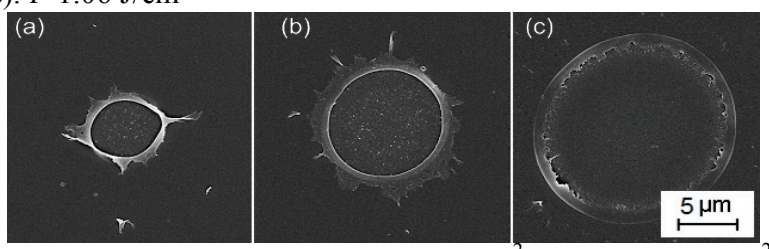

Frontside irradiation: (a). $\mathrm{f}=0.46 \mathrm{~J} / \mathrm{cm}^{2} ;$ (b). $\mathrm{f}=0.55 \mathrm{~J} / \mathrm{cm}^{2}$; (c). $\mathrm{f}=1.36 \mathrm{~J} / \mathrm{cm}^{2}$

Fig 5. Single pulse ablation induced by $355 \mathrm{~nm}$ from the backside (upper) and frontside (bottom) irradiation.

For $1064 \mathrm{~nm}$ pulse, the ablation results revealed that the PEDOT:PSS material could not be completely removed in the low fluence region shown in figure 6 . No apparent difference can be observed for both directions. High laser fluence leads to complete removal of the material. But with Gaussian beam profile, a large area of modified material was resided around the spot, especially for the frontside illumination case. Also the rim quality is poorer compared to using $355 \mathrm{~nm}$ wavelength pulses.

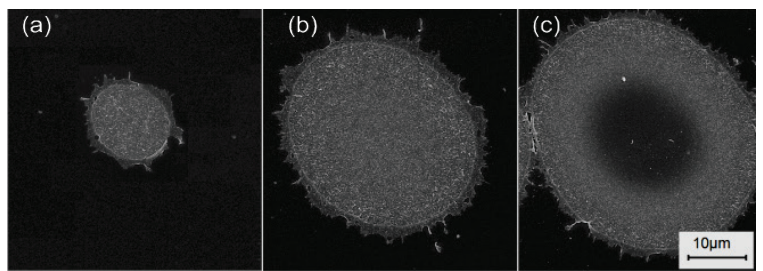

Backside irradiation: (a). $\mathrm{f}=0.11 \mathrm{~J} / \mathrm{cm}^{2} ;$ (b). $\mathrm{f}=0.42 \mathrm{~J} / \mathrm{cm}^{2}$; (c). $\mathrm{f}=0.92 \mathrm{~J} / \mathrm{cm}^{2}$

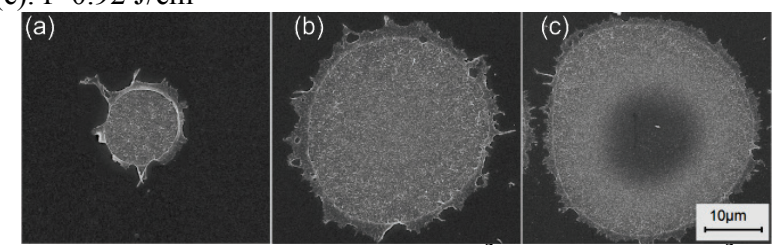

Frontside irradiation: (a). $\mathrm{f}=0.11 \mathrm{~J} / \mathrm{cm}^{2}$; (b). $\mathrm{f}=0.42 \mathrm{~J} / \mathrm{cm}^{2}$; (c). $\mathrm{f}=0.92 \mathrm{~J} / \mathrm{cm}^{2}$

Fig 6. The single pulse ablation induced by $1064 \mathrm{~nm}$ from the backside (upper) and frontside (bottom) irradiation.

A series of ablation experiments with variation of laser energy was conducted in order to derive the ablation thresholds. By measuring the diameter of the ablated spots using white-light interferometer we were able to plot them against the laser fluence (figure 7). The ablation thresholds can be achieved by extrapolating the squared diameter to 0 . For $100 \mathrm{~nm}$ thick PEDOT:PSS film, the ablation thresholds of $1064 \mathrm{~nm}$ wavelength are about $0.057 \mathrm{~J} / \mathrm{cm}^{2}$ and 0.082 $\mathrm{J} / \mathrm{cm}^{2}$ for frontside and backside illumination, respectively. For the $355 \mathrm{~nm}$ wavelength, the thresholds are $0.36 \mathrm{~J} / \mathrm{cm}^{2}$ and $0.33 \mathrm{~J} / \mathrm{cm}^{2}$ for frontside and backside ablation, respectively. Table 1 listed the critical laser fluence thresholds for initial PEDOT:PSS film ablation and glass substrate damage. It is clear that the ablation threshold for the $1064 \mathrm{~nm}$ wavelength is lower than the one for $355 \mathrm{~nm}$ because of the higher absorption. However, there is no significant difference in the ablation threshold for frontside and backside illumination using the uv wavelength.

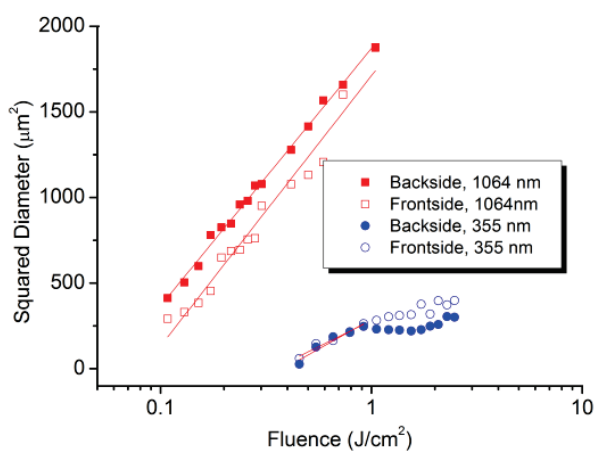

Fig 7. Single pulse ablation spot size varies with the pulse fluence. The ablation area increases faster for $1064 \mathrm{~nm}$ as the increasing of incident laser fluence.

\begin{tabular}{|l|l|l|l|l|}
\hline \multirow{2}{*}{$\left(\mathrm{J} / \mathrm{cm}^{2}\right)$} & \multicolumn{2}{|c|}{$1064 \mathrm{~nm}$} & \multicolumn{2}{c|}{$355 \mathrm{~nm}$} \\
\cline { 2 - 5 } & frontside & backside & frontside & backside \\
\hline PEDOT:PSS & 0.082 & 0.057 & 0.358 & 0.324 \\
\hline Substrate & $>1.1$ & $>1$ & $>2.4$ & 0.919 \\
\hline
\end{tabular}

Table 1. Summary of the critical thresholds to initialize the PEDOT:PSS film ablation and the glass damage.

The shoulder height of the spot rim is always undesirable in practical applications and should be as low as possible, which is mainly resulting from the Gaussian profile of the energy distribution of the laser beam. At the rim of the spot the energy is lower than in the center, which will introduce the shoulder when the laser fluence is lower than the removing threshold. From the SEM pictures we can see the shoulder comes from the film punched out from the spot center. This observation supports the thesis that a photomechanical force should be the main driver for ablation of PEDOT:PSS film for both wavelengths. Figure 8(a) shows the results for the $355 \mathrm{~nm}$ wavelength. It can be seen that with frontside illumination the shoulder height is significantly lower. Also higher laser fluence could lead to better quality. Figure 8(b) illustrates the case for the fundamental wavelength. The shoulder height is higher when irradiated from the backside. In summary, the lowest shoulder height is obtained using frontside illumination and pulses in the uv wavelength range. The value could be decreased to below $50 \mathrm{~nm}$. 

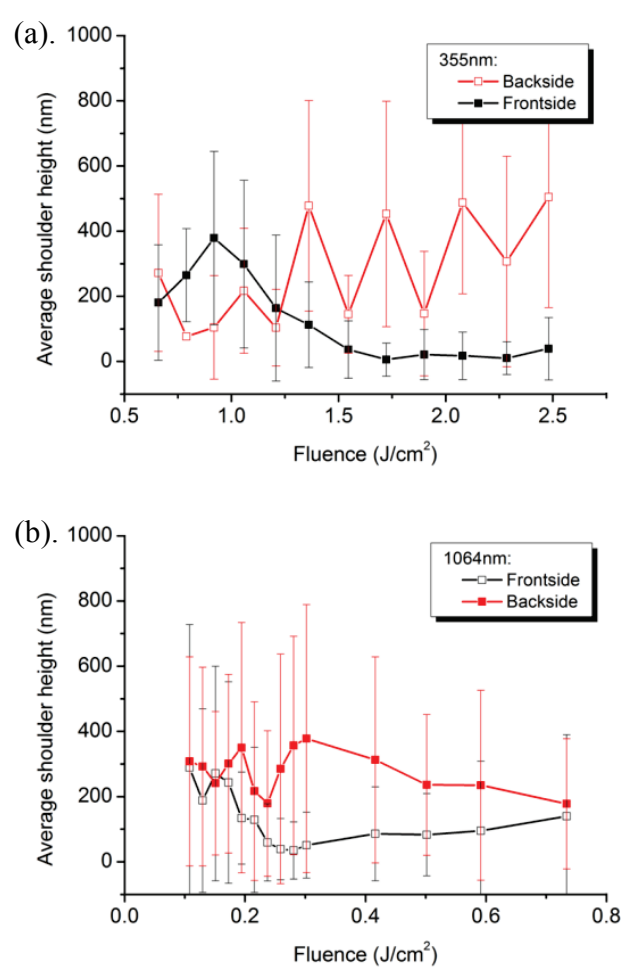

Fig 8. The average shoulder height varies with the pulse fluence. (a). $355 \mathrm{~nm}$ wavelength; (b). $1064 \mathrm{~nm}$ wavelength.

As mentioned above the film thickness is important for different applications. Therefore determining the ablation threshold for each thickness becomes critical. Figure 9 demonstrates the results for both wavelengths from frontside illumination. For the $355 \mathrm{~nm}$ case, the ablation threshold changes periodically as indicated by the guided line. When the film thickness decreases to be comparable to the laser wavelength of $355 \mathrm{~nm}$, an effective interference occurs by the reflective beams from the upper and lower boundaries of film interface which governs the amount of absorbed laser energy by the film. The constructive and destructive interferences modulate the absorbed energy periodically according to the film thickness by $\lambda / 2 n_{f}$ for vertically incident light, where $n_{f}$ is the refractive index of the film material. At $355 \mathrm{~nm}$ wavelength the refractive index is $n_{f}=1.5$ and the period $\lambda / 2 n_{f}=118 \mathrm{~nm}$ is nearly the same as measured from figure 9 (a) [11]. For the $1064 \mathrm{~nm}$ wavelength, the refractive index is $n_{f}=1.3$ resulting in $2 n_{f} d / \lambda<1$ for film thicknesses smaller than $400 \mathrm{~nm}$, effective interference cannot be achieved in this region. As the film becomes very thin, the heated volume of the film decreases linearly with film thickness $d$, reducing faster than the absorbed laser energy decreasing $\left(1-e^{-\alpha d}\right)$. If both boundaries of the film are isolated, the ablation thresholds will decrease and converge with decreasing thickness [12]. However, due to the increasing energy losses into the substrate with thinner films, more laser energy is required leading to increasing ablation thresholds. As demonstrated in figure 9 (b), the ablation threshold increases dramatically when the film thickness becomes smaller than $180 \mathrm{~nm}$. For the film thicker than this critical thickness, the thermal diffusion loss from the surface into the film stays constant and the energy loss to the substrate could be neglected. Therefore the ablation threshold keeps almost constant, about $0.04 \mathrm{~J} / \mathrm{cm}^{2}$, which is equal to the value for the bulk material.
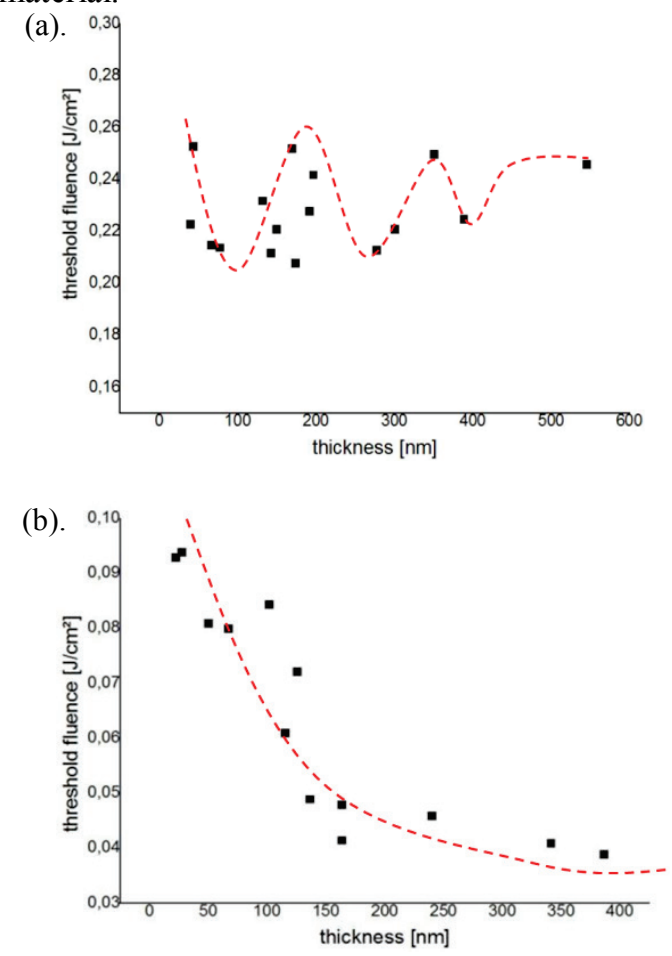

Fig 9. The ablation threshold varies with the film thickness. (a). $355 \mathrm{~nm}$; (b). $1064 \mathrm{~nm}$. For $355 \mathrm{~nm}$ wavelength the ablation thresholds changes periodically due to the formation of interference from the reflective beams by the film boundary interfaces. For $1064 \mathrm{~nm}$ wavelength the ablation thresholds decreased for the thick film because the film absorbed more laser energy but lost fewer energy into the substrate.

\subsection{Multi-pulse experiments}

Figure 10 shows the results from multi-pulse shots at the same position by 1 pulse, 3 pulses, 7 pulses and 9 pulses, respectively. For the $355 \mathrm{~nm}$ case, the ablation spot has become sharper after several lasers pulses. For the 1064 nm case, no apparent change was observed.

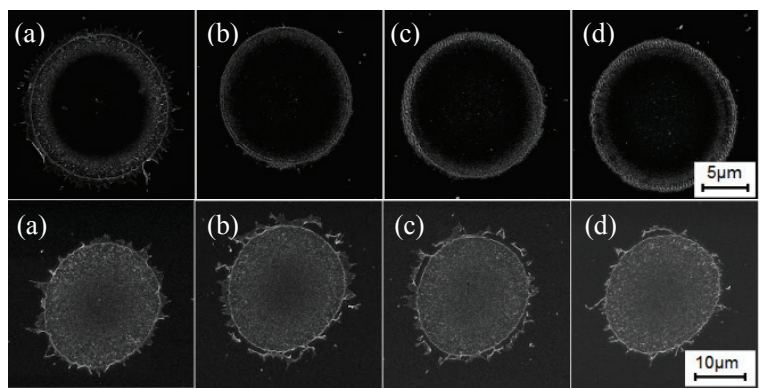

Fig 10. Multi-pulse ablation induced by $355 \mathrm{~nm}$ wavelength (upper) and $1064 \mathrm{~nm}$ wavelength (bottom). (a). 1 pulse; (b). 3 pulses; (c). 7 pulses; (d). 9 pulses. All ablation experiments have been conducted by frontside illumination.

For laser ablation of a line or an area, usually multi pulses ablation process is conducted with certain overlapping rate. Different overlapping rates could affect the morphology of the line. Figure 11 shows the results with $50 \%$ overlap (left) and $80 \%$ overlap (right) for frontside and backside illumination applying $355 \mathrm{~nm}$ pulses. 
It is obvious that the frontside patterning line is superior to the backside illumination one. High overlap ratios result in higher quality ablation areas than low overlapping rate. Figure 12 shows the similar experiments with fundamental wavelength pulses. The higher overlapping rate of $95 \%$ (frontside patterning) makes high quality lines compared to lower overlapping rates of $50 \%$. But the film cannot be removed completely at this wavelength.
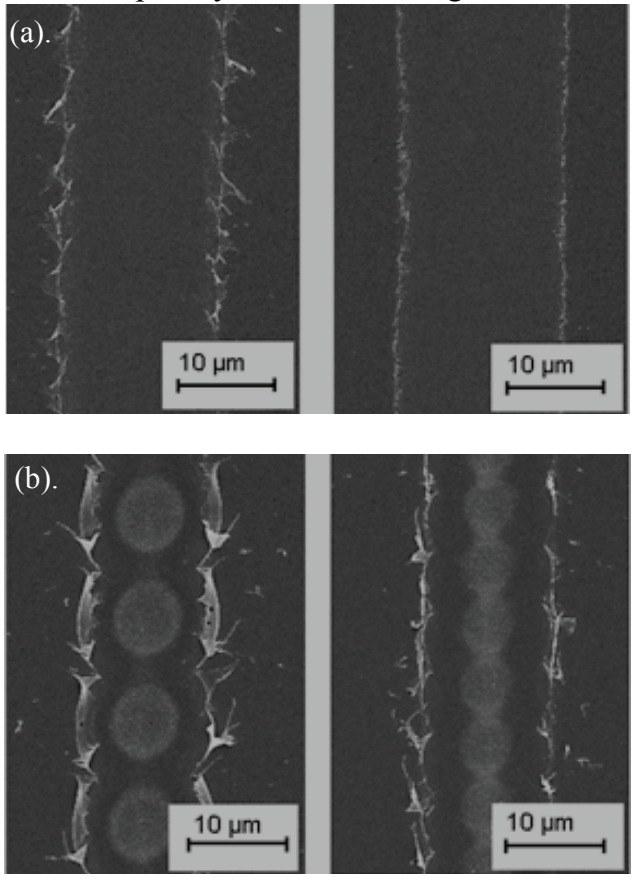

Fig 11. Ablated lines for the $355 \mathrm{~nm}$ wavelength pulses applying (a). frontside; (b). backside illumination. Both experiments were carried out with 50\% (left) and 80\% (right) overlapping rates and $1.36 \mathrm{~J} / \mathrm{cm}^{2}$ pulse fluence.
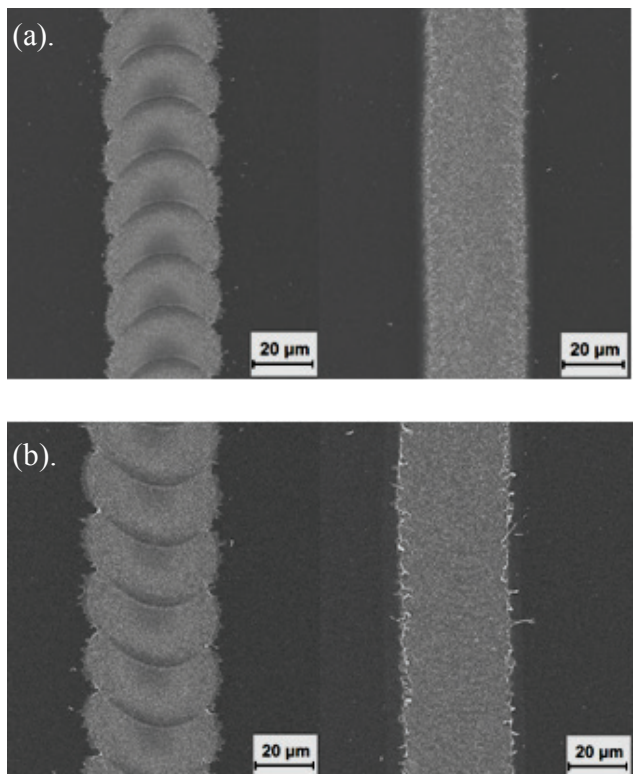

Fig 12. Ablation lines with $1064 \mathrm{~nm}$ pulse by (a). frontside; (b). backside. Both with 50\% (left) and 95\% (right) overlapping rates and $0.73 \mathrm{~J} / \mathrm{cm}^{2}$ pulse fluence.

It is clearly visible that the $355 \mathrm{~nm}$ wavelength pulse provides higher quality ablation lines. In figure 13 different overlapping rates were applied to investigate the shoulder height of the ablation lines. If the overlapping rate is higher than $60 \%$, the shoulder height tends to become constant. From experimental observation it can be seen that taking the ablation efficiency (low overlapping rate gives faster ablation speed) into account $355 \mathrm{~nm}$ wavelength pulses with a fluence of $1.36 \mathrm{~J} / \mathrm{cm}^{2}$ and $80 \%$ overlapping rate and frontside ablation is regarded to be the best result.

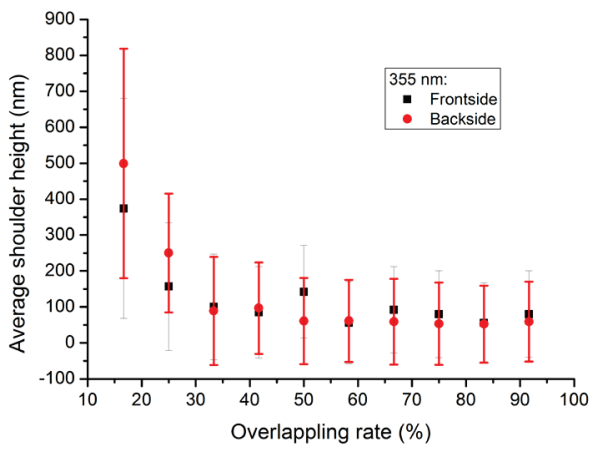

Fig 13. The shoulder height of the ablated lines by $355 \mathrm{~nm}$ wavelength pulses with different overlapping rate. The applied laser fluence was $1.36 \mathrm{~J} / \mathrm{cm}^{2}$ for both directions.

\section{Conclusion}

Picosecond laser with $355 \mathrm{~nm}$ and $1064 \mathrm{~nm}$ wavelengths pulses are used to patterning PEDOT:PSS thin film coated on glass substrate. The pulse irradiation from frontside and backside are compared. The ablation thresholds for both irradiation directions are presented. The frontside patterning method gives a super result, lower shoulder height and clearer patterning spots. The dependence of the ablation thresholds on the film thickness for both wavelengths is investigated. Multi pulses ablation and lines patterning experiment are also conducted. With optimized laser fluence $1.36 \mathrm{~J} / \mathrm{cm}^{2}$ and $80 \%$ overlapping rate, clear patterning line are obtained by $355 \mathrm{~nm}$ pulse from the frontside ablation.

\section{References}

[1] A. Wille, A. Drost, G. Klink, K. Bock, LOPE-C09 (2009).

[2] P. Cosseddu, A. Bonfiglio, Appl. Phys. Lett. 88, (2005)023506.

[3] T. Granlund, T. Nyberg, L. StolzRoman, M. Svensson, O. Inganäs, Adv. Mater., 12(4), (2000)269-273.

[4] F. Zhang, T. Nyberg, O. Inganäs, Nano. Lett. 12(2), (2002)1373-1377.

[5] A. Colsmann, F. Stenzela, G. Balthasara, H. Dao, U. Lemmera, Thin Solid Films, 517(5), (2009)1750-1752.

[6] B. Thomas, A. P. Alloncle, P. Delaporte, M. Sentis, S. Sanaur, M. Barret, P. Collot, Appl. Surf. Sci., 254(4), (2007)1206-1210.

[7] M. Schaefer, J. Holtkamp, A. Gillner, Synthetic Metal, 161(11-12), (2011)1051-1057.

[8] N. G. Semaltianos, C. Koidis, C. Pitsalidis, P. Karagiannidis, S. Logothetidis, W. Perrie, D. Liu, S. P. 
Edwardson, E. Fearon, R. J. Potter, Synthetic Metal, 161(5-6), (2011)431-439.

[9] A. F. Lasagni, J. L. Hendricks, C. M. Shaw, D. Yuan, D. C. Martin, S. Das, Appl. Surf. Sci., 255(22), (2009) 9186-9192.

[10] Y. Kima, A. M. Ballantyne, J. Nelson, D. C. Bradley, Organic Electronics, 10(1), (2009)205-209.

[11] L. A. A. Pettersson, S. Ghosh, O. Inganäs, Organic Electronics, 3(3-4), (2002)143-148.

[12] S. J. Henley, J. D. Carey, S. R. P. Silva, Phys. Rev. B, 72(19), (2005)195408.

(Received: June 17, 2011, Accepted: December 07, 2011) 